\title{
STABLE MINIMAL HYPERSURFACES IN THE HYPERBOLIC SPACE
}

\author{
Kеомkyo SeO
}

\begin{abstract}
In this paper we give an upper bound of the first eigenvalue of the Laplace operator on a complete stable minimal hypersurface $M$ in the hyperbolic space which has finite $L^{2}$-norm of the second fundamental form on $M$. We provide some sufficient conditions for minimal hypersurface of the hyperbolic space to be stable. We also describe stability of catenoids and helicoids in the hyperbolic space. In particular, it is shown that there exists a family of stable higher-dimensional catenoids in the hyperbolic space.
\end{abstract}

\section{Introduction}

In [6], Cheng, Li, and Yau derived comparison theorems for the first eigenvalue of Dirichlet boundary problem on any compact domain in minimal submanifolds of the hyperbolic space by estimating the heat kernel of the compact domain. Recall that the first eigenvalue $\lambda_{1}$ of a complete non-compact Riemannian manifold $M$ is defined by $\lambda_{1}=\inf _{\Omega} \lambda_{1}(\Omega)$, where the infimum is taken over all compact domains in $M$. Throughout this paper, we shall denote by $\mathbb{H}^{n}$ the $n$-dimensional hyperbolic space of constant sectional curvature -1 . Recently Candel [2] gave an upper bound for the first eigenvalue of the universal cover of a complete stable minimal surface in $\mathbb{H}^{3}$. Indeed, he proved:

Theorem ([2]). Let $\Sigma$ be a complete simply connected stable minimal surface in the 3-dimensional hyperbolic space. Then the first eigenvalue of $\Sigma$ satisfies

$$
\frac{1}{4} \leq \lambda_{1}(\Sigma) \leq \frac{4}{3} .
$$

In Section 2, we extend this theorem to simply connected stable minimal surfaces in a Riemannian manifold whose sectional curvature is bounded below and above by negative constants (Theorem 2.1). For a complete stable minimal

Received September 2, 2009.

2010 Mathematics Subject Classification. 53C40, 53C42.

Key words and phrases. stable minimal hypersurface, hyperbolic space, first eigenvalue.

This research was supported by the Sookmyung Women's University Research Grants 2010

(C)2011 The Korean Mathematical Society 
hypersurface $M$ in $\mathbb{H}^{n+1}$, Cheung and Leung [8] proved that

$$
\frac{1}{4}(n-1)^{2} \leq \lambda_{1}(M) .
$$

Here this inequality is sharp because equality holds when $M$ is totally geodesic ([12]). In this paper, it is proved that if $M$ is a complete stable minimal hypersurface in $\mathbb{H}^{n+1}$ with finite $L^{2}$-norm of the second fundamental form $A$, then we have (Theorem 2.2)

$$
\lambda_{1}(M) \leq n^{2} .
$$

Recall that a minimal hypersurface is called stable if the second variation of its volume is always nonnegative for any normal variation with compact support. More precisely, an $n$-dimensional minimal hypersurface $M$ in a Riemannian manifold $N$ is called stable if it holds that for any compactly supported Lipschitz function $f$ on $M$

$$
\int_{M}|\nabla f|^{2}-\left(|A|^{2}+\overline{\operatorname{Ric}}(\nu, \nu)\right) f^{2} d v \geq 0
$$

where $\nu$ is the unit normal vector of $M, \overline{\operatorname{Ric}}(\nu, \nu)$ denotes the Ricci curvature of $N$ in the $\nu$ direction, $|A|^{2}$ is the square length of the second fundamental form $A$, and $d v$ is the volume form for the induced metric on $M$. Note that when $N=\mathbb{H}^{n+1}, \overline{\operatorname{Ric}}(\nu, \nu)$ is equal to $-n$.

In Section 3, we give some conditions for complete minimal hypersurfaces in $\mathbb{H}^{n+1}$ to be stable as follows. If the $L^{\infty}$-norm of the second fundamental form is sufficiently small at every point in a complete minimal hypersurface $M$, then $M$ is stable (Theorem 3.1). Moreover if the $L^{n}$-norm of the second fundamental form is sufficiently small, then $M$ is stable (Theorem 3.2).

In 1981, Mori [13] explicitly described a one-parameter family of complete stable minimal rotation surfaces in $\mathbb{H}^{3}$. This example shows that a theorem due to do Carmo and Peng [4] and Fischer-Colbrie and Schoen [10] which says that a complete stable minimal surface in $\mathbb{R}^{3}$ must be a plane, does not hold in $\mathbb{H}^{3}$. Two years later, do Carmo and Dajczer [3] found a larger family of complete minimal rotation surfaces which are also stable. In Section 4, we study stability of catenoids in the hyperbolic space. In [3], it was shown that there exist a one-parameter family of unstable catenoids $M_{a}$ in $\mathbb{H}^{3}$ for $1 / 2<a<0.69$. We improve the upper bound of $a$ by estimating the $L^{2}$-norm of $|\nabla| A||$ in terms of the $L^{2}$-norm of the second fundamental form $A$ (Theorem 4.1). We also prove that the above unstable catenoid in $\mathbb{H}^{3}$ should have index one (Theorem 4.3). Recall that for a compact subset $\Omega$ in a complete minimal hypersurface $M$ in $\mathbb{H}^{n+1}$, the index of $\Omega$ is defined to be the number of negative eigenvalues of the stability operator $L:=\Delta-|A|^{2}+n$ on $\Omega$, counting the multiplicity. The index of $M$ is defined as the infimum of $\operatorname{Index}(\Omega)$ for all compact subset $\Omega$. Moreover we provide a family of complete minimal hypersurfaces in $\mathbb{H}^{n+1}$, which is an extension of Mori's result to higher dimensional cases (Theorem 4.4). Finally we investigate stability of helicoids in $\mathbb{H}^{3}$ in Section 5 . 


\section{First eigenvalue estimates}

In this section we first extend Candel's result to a simply connected complete minimal surface in a Riemannian manifold. The proof is actually based on Candel's proof.

Theorem 2.1. Let $\Sigma$ be a simply connected stable minimal surface in a 3 dimensional simply connected Riemannian manifold $N^{3}$ with sectional curvature $K_{N}$ satisfying $-b^{2} \leq K_{N} \leq-a^{2}<0$ for $0<a \leq b$. Then the first eigenvalue of $\Sigma$ satisfies

$$
\frac{1}{4} a^{2} \leq \lambda_{1}(\Sigma) \leq \frac{4}{3} b^{2}
$$

Proof. First we find an upper bound for $\lambda_{1}(\Sigma)$. Let $\left\{e_{1}, e_{2}, e_{3}\right\}$ be orthonormal frames in $N$ such that the vectors $\left\{e_{1}, e_{2}\right\}$ are tangent to $M$ and $e_{3}$ is normal to $M$. The Gauss curvature equation implies that the sectional curvature $K_{\Sigma}$ of $\Sigma$ satisfies

$$
\begin{aligned}
K_{\Sigma} & =R_{212}^{1}+h_{11} h_{22}-h_{12}^{2} \\
& =R_{212}^{1}-\frac{|A|^{2}}{2} \leq-a^{2}-\frac{|A|^{2}}{2}<0
\end{aligned}
$$

where $R_{212}^{1}$ is the sectional curvature of $N$ for the section determined by $e_{1}, e_{2}$ and $h_{i j}=\left\langle\bar{\nabla}_{e_{i}} e_{3}, e_{j}\right\rangle, \bar{\nabla}$ denoting Riemannian connection of $N$. Since $\Sigma$ is simply connected and has negative Gaussian curvature, there are global polar coordinates about any point in $\Sigma$. Using this polar coordinates, the metric tensor $g$ of $\Sigma$ can be written as

$$
g=d r^{2}+\phi(r, \theta)^{2} d \theta^{2}
$$

where $\phi(0, \theta)=0$ and $\left.\frac{\partial \phi}{\partial r}\right|_{(0, \theta)}:=\phi_{r}(0, \theta)=1$.

Using the equality $(2.1)$ and $\overline{\operatorname{Ric}}\left(e_{3}\right)=R_{131}^{3}+R_{232}^{3}$, the stability inequality (1.2) becomes

$$
\begin{aligned}
0 & \leq \int_{\Sigma}|\nabla f|^{2}-\left(|A|^{2}+\overline{\operatorname{Ric}}\left(e_{3}\right)\right) f^{2} d v \\
& \leq \int_{\Sigma}|\nabla f|^{2}-\left(R_{131}^{3}+R_{232}^{3}+2 R_{212}^{1}-2 K_{\Sigma}\right) f^{2} d v \\
& \leq \int_{\Sigma}|\nabla f|^{2}+2 K_{\Sigma} f^{2}+4 b^{2} f^{2} d v
\end{aligned}
$$

Since the inequality (2.2) holds for all compactly supported Lipschitz function $f$ on $\Sigma$, we shall choose some specific functions which depend only on the distance $r$ to the origin of the polar coordinates in $\Sigma$. More precisely, given $R>0$, we consider a family $\mathcal{F}$ of radial functions $f$ such that $f(0)=0, f(r)=0$ for $r \geq R>0$ and $f(r)$ is piecewise linear in $r$, that is, $f^{\prime \prime}(r)=0$ except for finitely many values of $r$. Note that the Gaussian curvature $K_{\Sigma}=-\frac{\phi_{r r}}{\phi}$. Thus 
the inequality $(2.2)$ can be written as

$$
2 \int_{0}^{2 \pi} \int_{0}^{R} f^{2} \phi_{r r} d r d \theta \leq \int_{0}^{2 \pi} \int_{0}^{R} f_{r}^{2} \phi d r d \theta+4 b^{2} \int_{0}^{2 \pi} \int_{0}^{R} f^{2} \phi d r d \theta .
$$

Integrating the left side of the above inequality twice by parts and using the properties of the function $f$, we obtain

$$
\begin{aligned}
-\int_{\Sigma} K_{\Sigma} f^{2} d v & =\int_{0}^{2 \pi} \int_{0}^{R} f^{2} \phi_{r r} d r d \theta \\
& =\int_{0}^{2 \pi}\left[f^{2} \phi_{r}\right]_{0}^{R} d \theta-2 \int_{0}^{2 \pi} \int_{0}^{R} f f_{r} \phi_{r} d r d \theta \\
& =\int_{0}^{2 \pi}\left[-2 f f_{r} \phi\right]_{0}^{R} d \theta+2 \int_{0}^{2 \pi} \int_{0}^{R}\left(f f_{r}\right)_{r} \phi d r d \theta \\
& =2 \int_{0}^{2 \pi} \int_{0}^{R}\left(f_{r}^{2} \phi+f f_{r r}\right) \phi d r d \theta \\
& =2 \int_{0}^{2 \pi} \int_{0}^{R} f_{r}^{2} \phi d r d \theta=2 \int_{\Sigma}|\nabla f|^{2} d v .
\end{aligned}
$$

Combining the inequality (2.2) with the equation (2.3), we get

$$
3 \int_{\Sigma}|\nabla f|^{2} d v \leq 4 b^{2} \int_{\Sigma} f^{2} d v
$$

Hence it follows that

$$
\lambda_{1}(\Sigma) \leq \inf _{f \in \mathcal{F}} \frac{\int_{\Sigma}|\nabla f|^{2} d v}{\int_{\Sigma} f^{2} d v} \leq \frac{4}{3} b^{2}
$$

Now we estimate a lower bound of $\lambda_{1}(\Sigma)$. The Laplacian of the distance function $r$ on $\Sigma \subset N$ satisfies [9]

$$
\Delta r \geq a\left(2-|\nabla r|^{2}\right) \operatorname{coth} a r \geq a .
$$

Integrating both sides over $\Omega \subset \Sigma$, we get

$$
a \operatorname{Area}(\Omega) \leq \int_{\Omega} \Delta r d v=\int_{\partial \Omega} \frac{\partial r}{\partial \nu} d s \leq \operatorname{Length}(\partial \Omega) .
$$

Recall that the Cheeger constant of a Riemannian manifold $M, h(M)$ is defined by

$$
h(M):=\inf _{\Omega} \frac{\operatorname{Length}(\partial \Omega)}{\operatorname{Area}(\Omega)},
$$

where $\Omega$ ranges over all open submanifold of $M$, with compact closure in $M$, and smooth boundary. Then applying Cheeger's inequality [5] and inequality (2.5), we obtain

$$
\lambda_{1}(\Sigma) \geq \frac{1}{4} h(\Sigma)^{2}=\frac{1}{4} a^{2} .
$$

Therefore the theorem follows from (2.4) and (2.6). 
The first eigenvalue of a complete minimal hypersurface in the hyperbolic space is bounded below by a constant $\frac{(n-1)^{2}}{4}$ as mentioned in the introduction. We give an upper bound for a stable minimal hypersurface with finite $L^{2}$-norm of the second fundamental form of $M$.

Theorem 2.2. Let $M$ be a complete stable minimal hypersurface in $\mathbb{H}^{n+1}$ with $\int_{M}|A|^{2} d v<\infty$. Then we have

$$
\frac{(n-1)^{2}}{4} \leq \lambda_{1}(M) \leq n^{2}
$$

Remark. There is no nontrivial example of such complete minimal hypersurfaces in $\mathbb{R}^{n+1}$, since do Carmo and Peng [4] proved that a complete stable minimal hypersurface $M$ in $\mathbb{R}^{n+1}$ with $\int_{M}|A|^{2} d v<\infty$ must be a hyperplane. However, there exist several examples of complete minimal hypersurfaces with finite $L^{2}$-norm of the second fundamental form in the hyperbolic space as we will see in Sections 4 and 5 . Note that we do not assume that $M$ is simply connected, which is different from Candel's result.

Proof. It is sufficient to show that $\lambda_{1}(M) \leq n^{2}$ by the inequality (1.1).

Take a function $f$ as follows. For a fixed point $p \in M$ and a fixed $R>0$, define a function $f: M \rightarrow \mathbb{R}$ by

$$
f(x)= \begin{cases}1, & r(x) \leq R \\ 2-\frac{r(x)}{R}, & R \leq r(x) \leq 3 R \\ -1, & 3 R \leq r(x) \leq 4 R \\ -5+\frac{r(x)}{R}, & 4 R \leq r(x) \leq 5 R \\ 0, & r(x) \geq 5 R\end{cases}
$$

where $r(x)$ is the distance from $p$ to $x$ in $M$. Then it follows that $\int_{M} f<0$. For $0 \leq t \leq R$, we now consider a family of functions $\left\{f_{t}\right\}$ defined by

$$
f_{t}(x)= \begin{cases}1, & r(x) \leq R, \\ 2-\frac{r(x)}{R}, & R \leq r(x) \leq 2 R+t, \\ -\frac{t}{R}, & 2 R+t \leq r(x) \leq 4 R+t, \\ -5+\frac{r(x)}{R}, & 4 R+t \leq r(x) \leq 5 R, \\ 0, & r(x) \geq 5 R .\end{cases}
$$

Then it is easy to see that there exists $t_{0}, 0<t_{0}<R$, such that $\int_{M} f_{t_{0}}=0$. From the definition of $\lambda_{1}(M)$ and $\lambda_{1}\left(B_{R}\right)$ for a ball $B_{R}$ of radius $R$ centered at $p$, it follows

$$
\lambda_{1}(M) \leq \lambda_{1}\left(B_{R}\right) \leq \frac{\int_{B_{R}}|\nabla \phi|^{2}}{\int_{B_{R}} \phi^{2}}
$$


for any compactly supported Lipschitz function $\phi$ satisfying $\int_{B_{R}} \phi=0$.

Now put $|A| f_{t_{0}}$ for $\phi$ in the inequality (2.7). Then

$$
\begin{aligned}
& \lambda_{1}(M) \int_{B_{R}}|A|^{2} f_{t_{0}}^{2} d v \\
\leq & \int_{B_{R}}\left|\nabla\left(|A| f_{t_{0}}\right)\right|^{2} d v \\
= & \int_{B_{R}}|\nabla| A||^{2} f_{t_{0}}^{2} d v+\int_{B_{R}}|A|^{2}\left|\nabla f_{t_{0}}\right|^{2} d v+2 \int_{B_{R}}|A| f_{t_{0}}\left\langle\nabla|A|, \nabla f_{t_{0}}\right\rangle d v .
\end{aligned}
$$

Moreover, using Schwarz inequality, for any positive number $\alpha>0$, we have

$$
2 \int_{B_{R}}|A| f_{t_{0}}\left\langle\nabla|A|, \nabla f_{t_{0}}\right\rangle d v \leq \alpha \int_{B_{R}}|A|^{2}\left|\nabla f_{t_{0}}\right|^{2} d v+\frac{1}{\alpha} \int_{B_{R}}|\nabla| A||^{2} f_{t_{0}}^{2} d v .
$$

Therefore we obtain

$$
\lambda_{1}(M) \int_{B_{R}}|A|^{2} f_{t_{0}}^{2} d v \leq\left(1+\frac{1}{\alpha}\right) \int_{B_{R}}|\nabla| A||^{2} f_{t_{0}}^{2} d v+(1+\alpha) \int_{B_{R}}|A|^{2}\left|\nabla f_{t_{0}}\right|^{2} d v .
$$

On the other hand, Chern, do Carmo, and Kobayashi [7] showed that

$$
\sum h_{i j} \Delta h_{i j}=-\sum h_{i j}^{2} h_{k l}^{2}-n \sum h_{i j}^{2} .
$$

Furthermore, we have

$$
|A| \Delta|A|+|\nabla| A||^{2}=\frac{1}{2} \Delta|A|^{2}=\sum h_{i j k}^{2}+\sum h_{i j} \Delta h_{i j} .
$$

Combining (2.9) with (2.10), we get

$$
|A| \Delta|A|+|A|^{4}+n|A|^{2}=|\nabla A|^{2}-|\nabla| A||^{2} .
$$

However the curvature estimate by Xin [16] says that

$$
|\nabla A|^{2}-|\nabla| A||^{2} \geq \frac{2}{n}|\nabla| A||^{2}
$$

and hence we have

$$
|A| \Delta|A|+|A|^{4}+n|A|^{2} \geq \frac{2}{n}|\nabla| A||^{2} .
$$

Multiplying both sides by a Lipschitz function $f^{2}$ with compact support in $B_{R} \subset M$ and integrating over $B_{R}$, we have

$$
\int_{B_{R}} f^{2}|A| \Delta|A| d v+\int_{B_{R}} f^{2}|A|^{4} d v+n \int_{B_{R}} f^{2}|A|^{2} d v \geq \frac{2}{n} \int_{B_{R}} f^{2}|\nabla| A||^{2} d v .
$$

The divergence theorem yields that

$$
\begin{aligned}
0 & =\int_{B_{R}} \operatorname{div}\left(|A| f^{2} \nabla|A|\right) d v \\
& =\int_{B_{R}} f^{2}|A| \Delta|A| d v+\int_{B_{R}}|\nabla| A||^{2} f^{2} d v+2 \int_{B_{R}}|A| f\langle\nabla|A|, \nabla f\rangle d v .
\end{aligned}
$$


Therefore

$$
\begin{array}{r}
\int_{B_{R}} f^{2}|A|^{4} d v+n \int_{B_{R}} f^{2}|A|^{2} d v-\int_{B_{R}}|\nabla| A||^{2} f^{2} d v-2 \int_{B_{R}}|A| f\langle\nabla|A|, \nabla f\rangle d v \\
\geq \frac{2}{n} \int_{B_{R}} f^{2}|\nabla| A||^{2} d v .
\end{array}
$$

Since $M$ is stable, we have

$$
\int_{M}|\nabla \phi|^{2}-\left(|A|^{2}-n\right) \phi^{2} d v \geq 0
$$

for any compactly supported function $\phi$ on $M$. Substituting $|A| f$ for $\phi$ gives

$$
\int_{B_{R}}|\nabla(|A| f)|^{2}-\left(|A|^{2}-n\right)|A|^{2} f^{2} d v \geq 0
$$

Thus

$$
\begin{array}{r}
\int_{B_{R}}|A|^{2}|\nabla f|^{2} d v+\int_{B_{R}}|\nabla| A||^{2} f^{2} d v+2 \int_{B_{R}}|A| f\langle\nabla|A|, \nabla f\rangle d v \\
\geq \int_{B_{R}}|A|^{4} f^{2} d v-n \int_{B_{R}}|A|^{2} f^{2} d v
\end{array}
$$

By (2.11) and (2.12), we obtain, for any compactly supported Lipschitz function $f$

$$
\int_{B_{R}}|A|^{2}|\nabla f|^{2} d v+2 n \int_{B_{R}}|A|^{2} f^{2} d v \geq \frac{2}{n} \int_{B_{R}}|\nabla| A||^{2} f^{2} d v .
$$

Combining (2.8) with the inequality obtained by substituting $f_{t_{0}}$ for $f$ in (2.13), we get

$$
\left\{1+\frac{2 n(1+\alpha)}{\lambda_{1}(M)}\right\} \int_{B_{R}}|A|^{2}\left|\nabla f_{t_{0}}\right|^{2} d v \geq\left.\left\{\frac{2}{n}-\frac{2 n\left(1+\frac{1}{\alpha}\right)}{\lambda_{1}(M)}\right\} \int_{B_{R}}|\nabla| A\right|^{2} f_{t_{0}}^{2} d v .
$$

Now suppose that $\lambda_{1}(M)>n^{2}$. Choosing $\alpha>0$ sufficiently large and letting $R \rightarrow \infty$, we obtain $\nabla|A| \equiv 0$, i.e., $|A|$ is constant. However, since $\int_{M}|A|^{2}<\infty$ and the volume of $M$ is infinite, it follows from the above inequality (2.14) that $|A| \equiv 0$ which means that $M$ is a totally geodesic hyperplane. Since the first eigenvalue of totally geodesic hyperplane in $\mathbb{H}^{n+1}$ is equal to $\frac{(n-1)^{2}}{4}$, this is a contradiction. Therefore we get $\lambda_{1}(M) \leq n^{2}$.

\section{Sufficient conditions for stability of minimal hypersurfaces in $\mathbb{H}^{n+1}$}

In this section we prove that if $|A|$ is bounded by a sufficiently small constant at every point in a complete minimal hypersurface $M$ in the hyperbolic space, then $M$ must be stable. More precisely,

Theorem 3.1. Let $M$ be a complete minimal hypersurface in $\mathbb{H}^{n+1}$. If $|A| \leq$ $\frac{(n+1)^{2}}{4}$ at every point in $M$, then $M$ is stable. 
Proof. Since the lower bound of the first eigenvalue of $M$ is $\frac{(n-1)^{2}}{4}$ by the inequality (1.1), we have

$$
\frac{(n-1)^{2}}{4} \leq \lambda_{1}(M) \leq \frac{\int_{M}|\nabla f|^{2}}{\int_{M} f^{2}}
$$

for every compactly supported Lipschitz function $f$ on $M$. Hence the assumption that $|A|^{2} \leq \frac{(n+1)^{2}}{4}$ implies

$$
\int_{M}|\nabla f|^{2}-\left(|A|^{2}-n\right) f^{2} d v \geq \int_{M}\left(\lambda_{1}(M)+n-|A|^{2}\right) f^{2} d v \geq 0
$$

which completes the proof.

It is well-known that the following Sobolev inequality [11] on a minimal hypersurface $M$ in $\mathbb{H}^{n+1}$ holds

$$
\left(\int_{M}|f|^{\frac{2 n}{n-2}} d v\right)^{\frac{n-2}{n}} \leq C_{s} \int_{M}|\nabla f|^{2} d v
$$

where $C_{s}$ is the Sobolev constant which dependent only on $n \geq 3$. Using this inequality one obtains another sufficient condition for minimal hypersurfaces to be stable.

Theorem 3.2. Let $M$ be a complete minimal hypersurface in $\mathbb{H}^{n+1}, n \geq 3$. If $\int_{M}|A|^{n} d v \leq\left(\frac{1}{C_{s}}\right)^{\frac{n}{2}}$, then $M$ is stable.

Proof. It suffices to show that

$$
\int_{M}|\nabla f|^{2}-\left(|A|^{2}-n\right) f^{2} d v \geq 0
$$

for all compactly supported Lipschitz function $f$. By Sobolev inequality (3.1), we have

$$
\int_{M}|\nabla f|^{2}-\left(|A|^{2}-n\right) f^{2} d v \geq \frac{1}{C_{s}}\left(\int_{M}|f|^{\frac{2 n}{n-2}} d v\right)^{\frac{n-2}{n}}-\int_{M}|A|^{2} f^{2} d v .
$$

On the other hand, applying Hölder inequality, we get

$$
\int_{M}|A|^{2} f^{2} d v \leq\left(\int_{M}|A|^{n} d v\right)^{\frac{2}{n}}\left(\int_{M}|f|^{\frac{2 n}{n-2}} d v\right)^{\frac{n-2}{n}} .
$$

Combining (3.2) with (3.3) we have

$$
\begin{aligned}
\int_{M}|\nabla f|^{2}-\left(|A|^{2}-n\right) f^{2} d v & \geq\left\{\frac{1}{C_{s}}-\left(\int_{M}|A|^{n} d v\right)^{\frac{2}{n}}\right\}\left(\int_{M}|f|^{\frac{2 n}{n-2}} d v\right)^{\frac{n-2}{n}} \\
& \geq 0, \quad \text { (by assumption) }
\end{aligned}
$$

which completes the proof. 


\section{Catenoids in $\mathbb{H}^{n+1}$}

In [3], do Carmo and Dajczer proved that there exist three types of rotationally symmetric minimal hypersurfaces in $\mathbb{H}^{n+1}$. Following [3], we say that a rotationally symmetric minimal hypersurface $M$ is a spherical catenoid, if $M$ is foliated by spheres, a hyperbolic catenoid, if it is foliated by hyperbolic spaces, and a parabolic catenoid, if it is foliated by horospheres. Do Carmo and Dajczer showed that the complete hyperbolic and parabolic catenoids in $\mathbb{H}^{3}$ are all globally stable. Furthermore they also proved that there exist some unstable spherical catenoids in $\mathbb{H}^{3}$. In what follows, we shall denote by $\mathbb{L}^{n+1}$ the space of $(n+1)$-tuples $x=\left(x_{1}, \ldots, x_{n+1}\right)$ with Lorentzian metric $\langle x, y\rangle=-x_{1} y_{1}+x_{2} y_{2}+\cdots+x_{n+1} y_{n+1}$, where $y=\left(y_{1}, \ldots, y_{n+1}\right)$. The hyperbolic space $\mathbb{H}^{n}$ is the simply connected hypersurface of $\mathbb{L}^{n+1}$ defined by $\mathbb{H}^{n}=\left\{x \in \mathbb{L}^{n+1}:\langle x, x\rangle=-1, x_{1} \geq 1\right\}$.

To state their result for unstable spherical catenoids in $\mathbb{H}^{3}$, we parametrize a spherical catenoid in $\mathbb{H}^{3}$ as follows (See [3] and [13]). For each constant $a>1 / 2$, define the mapping $f_{a}: \mathbb{R} \times \mathbb{S}^{1} \rightarrow \mathbb{H}^{3}$ by

$$
\begin{array}{r}
f_{a}(s, \theta)=\left(\sqrt{a \cosh (2 s)+\frac{1}{2}} \cosh \phi(s), \sqrt{a \cosh (2 s)+\frac{1}{2}} \sinh \phi(s),\right. \\
\left.\sqrt{a \cosh (2 s)-\frac{1}{2}} \cos \theta, \sqrt{a \cosh (2 s)-\frac{1}{2}} \sin \theta\right),
\end{array}
$$

where $\phi(s)=\left(a^{2}-\frac{1}{4}\right)^{1 / 2} \int_{0}^{s} \frac{1}{\left(a \cosh (2 t)+\frac{1}{2}\right)\left(a \cosh (2 t)-\frac{1}{2}\right)^{1 / 2}} d t$.

Do Carmo and Dajczer observed that if $1 / 2<a<c_{0}, c_{0} \simeq 0.69$, then the spherical catenoids $M_{a}$ 's are unstable. We shall improve the upper bound $c_{0}$ by using the inequality (2.13), which is different from their method. Letting $R \rightarrow \infty$ in (2.13), one can immediately obtain the following.

Theorem 4.1. Let $M$ be a complete stable minimal hypersurface in $\mathbb{H}^{n+1}$ with $\int_{M}|A|^{2} d v<\infty$. Then we have

$$
\int_{M}|\nabla| A||^{2} d v \leq n^{2} \int_{M}|A|^{2} d v
$$

and hence the $L^{2}$-norm of $|\nabla| A||$ is finite.

As a consequence of this Theorem 4.1, the upper bound $c_{0}$ due to do Carmo and Dajczer can be improved as follows.

Corollary 4.2. Spherical catenoid $M_{a}$ in $\mathbb{H}^{3}$ is unstable for $1 / 2<a<c_{0}, c_{0} \simeq$ 0.73 .

Proof. We first observe that the spherical catenoid $M_{a}$ satisfies $\int_{M_{a}}|A|^{2} d v<$ $\infty$. To see this, we note that for $a>1 / 2$

$$
I=d s^{2}+\left(a \cosh 2 s-\frac{1}{2}\right) d t^{2}
$$




$$
\begin{aligned}
|A|^{2} & =\frac{2\left(a^{2}-\frac{1}{4}\right)}{\left(a \cosh 2 s-\frac{1}{2}\right)^{2}}, \\
d v & =\left(a \cosh 2 s-\frac{1}{2}\right)^{\frac{1}{2}} d s d t \text { for } a>\frac{1}{2} \text { and } 0 \leq t \leq 2 \pi .
\end{aligned}
$$

Thus

$$
\begin{aligned}
\int_{M}|A|^{2} d v & =8 \pi\left(a^{2}-\frac{1}{4}\right) \int_{0}^{\infty} \frac{1}{\left(a \cosh 2 s-\frac{1}{2}\right)^{\frac{3}{2}}} d s \\
& <8 \pi\left(a^{2}-\frac{1}{4}\right) \int_{0}^{\infty} \frac{1}{\left(a+a s^{2}-\frac{1}{2}\right)^{\frac{3}{2}}} d s<\infty
\end{aligned}
$$

Now define a function $F(a)$ by

$$
F(a):=4 \int_{M}|A|^{2} d v-\int_{M}|\nabla| A||^{2} d v
$$

Using $|\nabla| A||=\left|-\sqrt{2\left(a^{2}-\frac{1}{4}\right)} \frac{2 a \sinh 2 s}{\left(a \cosh 2 s-\frac{1}{2}\right)^{2}}\right|$, we have

$$
F(a)=32 \pi\left(a^{2}-\frac{1}{4}\right) \int_{0}^{\infty}\left\{\frac{1}{\left(a \cosh 2 s-\frac{1}{2}\right)^{\frac{3}{2}}}-a^{2} \frac{\sinh ^{2} 2 s}{\left(a \cosh 2 s-\frac{1}{2}\right)^{\frac{7}{2}}}\right\} d s .
$$

By Theorem 4.1, we see that if $M_{a}$ is stable for some $a$, then $F(a) \geq 0$. However a straightforward computation by using a computer shows that $F(a)<0$ for $1 / 2<a<c_{0}, c_{0} \simeq 0.73$. Therefore we get the conclusion.

As we have seen before, there exist some unstable catenoids in $\mathbb{H}^{3}$. Hence it is interesting to find the index of such catenoids which measures the degree of instability. It is well-known that catenoids have index 1 in $\mathbb{R}^{3}$. Very recently, Tam and Zhou [15] proved that higher dimensional catenoids in $\mathbb{R}^{n+1}$ with $n \geq 3$ have index one. Motivated by this, we shall prove the following result using the similar arguments as in [15].

Theorem 4.3. Let $M$ be a spherical catenoid in $\mathbb{H}^{n+1}$. Then the index of $M$ is at most 1 .

Proof. We may assume that $M$ is unstable. It suffices to show that the second eigenvalue $\lambda_{2}(D) \geq 0$ of the stability operator $L=\Delta+|A|^{2}-n$ on some bounded domain $D \subset M$. We prove this theorem by contradiction. For this purpose, suppose that the index of $M$ is at least 2. Then there exists a domain $D(R)=(-R, R) \times \mathbb{S}^{n-1}$ such that $\lambda_{2}(D(R))<0$ for $R>0$.

Let $f$ be the second eigenfunction satisfying

$$
\begin{aligned}
L f & =-\lambda_{2}(D(R)) f \text { in } D(R) \\
f & =0 \text { on } \partial D(R) .
\end{aligned}
$$

We claim that $f$ is rotationally symmetric, that is, $f\left(t_{1}, \ldots, t_{n-1}, s\right)=f(s)$. 
To see this, consider a generating curve $\alpha(s):=(x(s), y(s), z(s), 0, \ldots, 0) \subset$ $\mathbb{H}^{n+1}$ and its rotation axis $\{(\cosh u, \sinh u, 0, \ldots, 0)\} \subset \mathbb{H}^{n+1}$. Let $P_{0}$ be the totally geodesic hyperplane such that $P_{0} \perp \alpha^{\prime}(0)$ and $\alpha(0)=(1,0, \ldots, 0) \in P_{0}$. For any vector $v \in S_{\alpha(0)} P_{0}:=\left\{v \in T_{\alpha(0)} P_{0}:|v|=1\right\}$, denote by $P_{v}$ the (unique) totally geodesic hyperplane such that $\alpha(0) \in P_{v}$ and $P_{v} \perp v$ at $\alpha(0)$.

Let $\sigma_{v}$ be the reflection across the hyperplane $P_{v}$. For any point $p \in D(R)$, define the difference function $\varphi_{v}\left(t_{1}, \ldots, t_{n-1}, s\right)$ by

$$
\varphi_{v}\left(t_{1}, \ldots, t_{n-1}, s\right):=f\left(t_{1}, \ldots, t_{n-1}, s\right)-f_{v}\left(t_{1}, \ldots, t_{n-1}, s\right),
$$

where $f_{v}(p):=f\left(\sigma_{v}(p)\right)$. Then it follows that $\Delta f=\Delta f_{v}$. Thus

$$
\left\{\begin{array}{l}
L \varphi_{v}=-\lambda_{2}(D(R)) \text { in } D(R) \\
\varphi_{v}=0 \text { on } \partial D(R) \cap P_{v} .
\end{array}\right.
$$

Since $P_{v}$ divides $D(R)$ into two parts, we choose one of them and denote by $D_{v}^{+}(R)$. Note that $D_{v}^{+}(R)$ is a minimal graph over a domain $P_{v}$. Hence $D_{v}^{+}(R)$ is stable. However from (4.1) and the assumption that $\lambda_{2}<0$, it follows that $\varphi_{v} \equiv 0$. As in the Euclidean space, any rotation around the axis $\{(\cosh u, \sinh u, 0, \ldots, 0)\} \subset \mathbb{H}^{n+1}$ can be expressed as a composition of finite number of reflections. Since $v$ was arbitrarily chosen, the claim is obtained.

On the other hand, since the second eigenfunction of the operator $L$ changes sign, there exists a number $r_{0} \in(-R, R)$ satisfying $f\left(r_{0}\right)=0$. We may assume that $r_{0} \geq 0$ and the second eigenfunction $f(s)>0$ on the domain $D\left(r_{0}, R\right)=\left\{\left(t_{1}, \ldots, t_{n-1}, s\right) \in D(R): s \in\left(r_{o}, R\right)\right\}$. The function $f$ is still an eigenfunction of $L$ on $D\left(r_{0}, R\right)$. Moreover it is easy to see that $D\left(r_{0}, R\right)$ is a minimal graph over the hyperplane $P_{0}$, which means that $D\left(r_{0}, R\right)$ is stable. This is a contradiction to the assumption that $\lambda_{2}<0$. Therefore we get the conclusion.

Remark. When $n=2$, we observed that a spherical catenoid $M_{a} \subset \mathbb{H}^{3}$ is unstable if $1 / 2<a<0.73$ in Corollary 4.2. It follows from the above theorem that these spherical catenoids must have index 1.

We now describe stability of hyperbolic catenoids in the hyperbolic space $\mathbb{H}^{n+1}$. For that purpose, we give a parametrization of a hyperbolic catenoid generated by a curve $(x(s), y(s), z(s))$ in the hyperbolic plane $\mathbb{H}^{2}$ which is parametrized by arclength. It follows that

$$
\begin{gathered}
-x(s)^{2}+y(s)^{2}+z(s)^{2}=-1, \quad x(s) \geq 1, \\
-x^{\prime}(s)^{2}+y^{\prime}(s)^{2}+z^{\prime}(s)^{2}=1, \\
f\left(t_{1}, \ldots, t_{n-1}, s\right)=\left(x(s) \varphi_{1}, \ldots, x(s) \varphi_{n}, y(s), z(s)\right), \\
\varphi_{i}=\varphi_{i}\left(t_{1}, \ldots, t_{n-1}\right), \quad-\varphi_{1}^{2}+\varphi_{2}^{2}+\cdots+\varphi_{n}^{2}=-1,
\end{gathered}
$$


where $\left(\varphi_{1}, \ldots, \varphi_{n}\right)$ is an orthogonal parametrization of the hyperbolic space $\mathbb{H}^{n-1}$. From (4.2) and (4.3), $y(s)$ and $z(s)$ are determined by

$$
\begin{aligned}
& y(s)=\sqrt{x(s)^{2}-1} \sin \phi(s), \\
& z(s)=\sqrt{x(s)^{2}-1} \cos \phi(s),
\end{aligned}
$$

where $\phi(s)=\int_{0}^{s} \frac{\sqrt{x^{2}-x^{\prime 2}-1}}{x^{2}-1} d t$.

Using minimality and rotationally symmetric property of a catenoid, one can see that the direction of the parameters are principal directions and the principal curvatures are given by

$$
\begin{array}{r}
\lambda_{1}=\cdots=\lambda_{n-1}=-\frac{\sqrt{x^{2}-x^{\prime 2}-1}}{x}, \\
\lambda_{n}=\frac{x^{\prime \prime}-x}{\sqrt{x^{2}-x^{\prime 2}-1}}=(n-1) \frac{\sqrt{x^{2}-x^{\prime 2}-1}}{x}
\end{array}
$$

(See [3, Proposition 3.2]). Furthermore we can write down an ordinary differential equation as follows [3, Lemma 3.15]:

$$
x^{\prime}=\sqrt{x^{2}-1-a^{2} x^{2(1-n)}}, \quad a=\text { const. }
$$

To find a unique solution of (4.5), we fix initial data as follows:

$$
\begin{aligned}
x(0) & =t \geq 1, \\
x^{\prime}(0) & =0 .
\end{aligned}
$$

Then from the initial data it follows that

$$
a=t^{n-1} \sqrt{t^{2}-1} \geq 0 .
$$

Moreover in order to have a nontrivial parametrization of a hyperbolic catenoid we see that $a>0$. Therefore for each constant $t>1$ the parametrization $f\left(t_{1}, \ldots, t_{n-1}, s\right)$ defines a hyperbolic catenoid $M_{t}$ in $\mathbb{H}^{n+1}$. We now state a our result about stability of hyperbolic catenoids in the hyperbolic space.

Theorem 4.4. Let $M_{t}$ be a family of hyperbolic catenoids in $\mathbb{H}^{n+1}$ defined as in (4.4). Then $M_{t}$ is a complete stable hypersurface in $\mathbb{H}^{n+1}$ for $1<t<$ $1+\frac{(n+1)^{2}}{4 n(n-1)}$.

Proof. Observe that

$$
\begin{array}{rlrl}
|A|^{2} & =\sum \lambda_{i}^{2}=(n-1) \lambda_{1}^{2}+\lambda_{n}^{2}=(n-1) \lambda_{1}^{2}+(n-1)^{2} \lambda_{1}^{2} \\
& =n(n-1) \lambda_{1}^{2} & \\
& =n(n-1) \frac{x^{2}-x^{2}-1}{x^{2}} & & (\text { by }(4.5)) \\
& =n(n-1) \frac{a^{2}}{x^{2 n}} & \quad(\text { by }(4.6))
\end{array}
$$


Since $x(s)$ is monotonically increasing by (4.5), we get $x(s) \geq x(0)=t>1$. Therefore $|A|^{2} \leq n(n-1)\left(t^{2}-1\right)$. The assumption on $t$ implies that $|A|^{2} \leq$ $\frac{(n+1)^{2}}{4}$. The conclusion follows from Theorem 3.1.

Remark. It is not hard to see that the family $\left\{M_{t}\right\}$ of hyperbolic catenoids in the above theorem satisfy $\int_{M_{t}}|A|^{2} d v<\infty$ by using $\sqrt{x^{2}-1-a^{2}}<x^{\prime}<\sqrt{x^{2}-1}$, which is obtained from the equality (4.5) and the fact that $x>1$.

\section{Helicoids in $\mathbb{H}^{3}$}

Let $l$ be a geodesic in $\mathbb{H}^{3}$. Let $\left\{\psi_{t}\right\}$ be the translation of distance $t$ along $l$ and let $\left\{\varphi_{t}\right\}$ be the rotation of angle $t$ around $l$. Given any $\alpha \in \mathbb{R}$, one can see that $\lambda=\left\{\lambda_{t}\right\}=\left\{\psi_{t} \circ \varphi_{\alpha t}\right\}$ is a one-parameter subgroup of isometries of $\mathbb{H}^{3}$ which is called a helicoidal group of isometries with angular pitch $\alpha$. A helicoid in $\mathbb{H}^{3}$ is a $\lambda$-invariant surface (See [14]). In 1989, Ripoll [14] proved that a helicoid $M_{\alpha}$ with angular pitch $|\alpha|<1$ is stable by showing that such $M_{\alpha}$ foliates $\mathbb{H}^{3}$. In this section, we improve the upper bound of angular pitch $|\alpha|$ by simple arguments.

A helicoid $M_{\alpha} \subset \mathbb{H}^{3} \subset \mathbb{L}^{4}$ can be written explicitly as follows [1] :

$$
X(s, t)=(\cosh s \cosh t, \sinh s \cosh t, \cos \alpha s \sinh t, \sin \alpha s \sinh t) .
$$

A little computation shows that the first and second fundamental forms of $M_{\alpha}$ are given by

$$
\begin{aligned}
I & =\left(\cosh ^{2} t+\alpha^{2} \sinh ^{2} t\right) d s^{2}+d t^{2}, \\
I I & =-2 \frac{\alpha}{\sqrt{\cosh ^{2} t+\alpha^{2} \sinh ^{2} t}} d s d t .
\end{aligned}
$$

Since $\cosh ^{2} t+\alpha^{2} \sinh ^{2} t \geq 1$, it follows

$$
|A|^{2}=\frac{\alpha^{2}}{\cosh ^{2} t+\alpha^{2} \sinh ^{2} t}+\frac{\alpha^{2}}{\left(\cosh ^{2} t+\alpha^{2} \sinh ^{2} t\right)^{3}} \leq 2 \alpha^{2} .
$$

The following theorem is an immediate consequence of Theorem 3.1.

Theorem 5.1. A helicoid $M_{\alpha}$ with angular pitch $|\alpha|^{2} \leq \frac{9}{8}$ is stable.

\section{References}

[1] J. Barbosa, M. Dajczer, and L. Jorge, Minimal ruled submanifolds in spaces of constant curvature, Indiana Univ. Math. J. 33 (1984), no. 4, 531-547.

[2] A. Candel, Eigenvalue estimates for minimal surfaces in hyperbolic space, Trans. Amer. Math. Soc. 359 (2007), no. 8, 3567-3575.

[3] M. do Carmo and M. Dajczer, Rotation hypersurfaces in spaces of constant curvature, Trans. Amer. Math. Soc. 277 (1983), no. 2, 685-709.

[4] M. do Carmo and C. K. Peng, Stable complete minimal hypersurfaces, Proceedings of the 1980 Beijing Symposium on Differential Geometry and Differential Equations, Vol. 1, 2, 3 (Beijing, 1980), 1349-1358, Science Press, Beijing, 1982.

[5] I. Chavel, Isoperimetric Inequalities, Cambridge Tracts in Mathematics, 145. Cambridge University Press, Cambridge, 2001. 
[6] S. Y. Cheng, P. Li, and S.-T. Yau, Heat equations on minimal submanifolds and their applications, Amer. J. Math. 106 (1984), no. 5, 1033-1065.

[7] S. S. Chern, M. do Carmo, and S. Kobayashi, Minimal submanifolds of a sphere with second fundamental form of constant length, 1970 Functional Analysis and Related Fields (Proc. Conf. for M. Stone, Univ. Chicago, Chicago, Ill., 1968) pp. 59-75 Springer, New York.

[8] L. F. Cheung and P. F. Leung, Eigenvalue estimates for submanifolds with bounded mean curvature in the hyperbolic space, Math. Z. 236 (2001), no. 3, 525-530.

[9] J. Choe, The isoperimetric inequality for minimal surfaces in a Riemannian manifold, J. Reine Angew. Math. 506 (1999), 205-214.

[10] D. Fischer-Colbrie and R. Schoen, The structure of complete stable minimal surfaces in 3-manifolds of nonnegative scalar curvature, Comm. Pure Appl. Math. 33 (1980), no. 2, 199-211.

[11] D. Hoffman and J. Spruck, Sobolev and isoperimetric inequalities for Riemannian submanifolds, Comm. Pure Appl. Math. 27 (1974), 715-727.

[12] H. P. McKean, An upper bound to the spectrum of $\Delta$ on a manifold of negative curvature, J. Differential Geometry 4 (1970), 359-366.

[13] H. Mori, Minimal surfaces of revolution in $\mathrm{H}^{3}$ and their global stability, Indiana Univ. Math. J. 30 (1981), no. 5, 787-794.

[14] J. Ripoll, Helicoidal minimal surfaces in hyperbolic space, Nagoya Math. J. 114 (1989), $65-75$.

[15] L.-F. Tam and D. Zhou, Stability properties for the higher dimensional catenoid in $\mathbb{R}^{n+1}$, Proc. Amer. Math. Soc. 137 (2009), no. 10, 3451-3461.

[16] Y. L. Xin, Bernstein type theorems without graphic condition, Asian J. Math. 9 (2005), no. $1,31-44$.

Department of Mathematics

SOOKMYUng WOMEN'S UNIVERSiTy

SeOUl 140-742, KoreA

E-mail address: kseo@sookmyung.ac.kr 